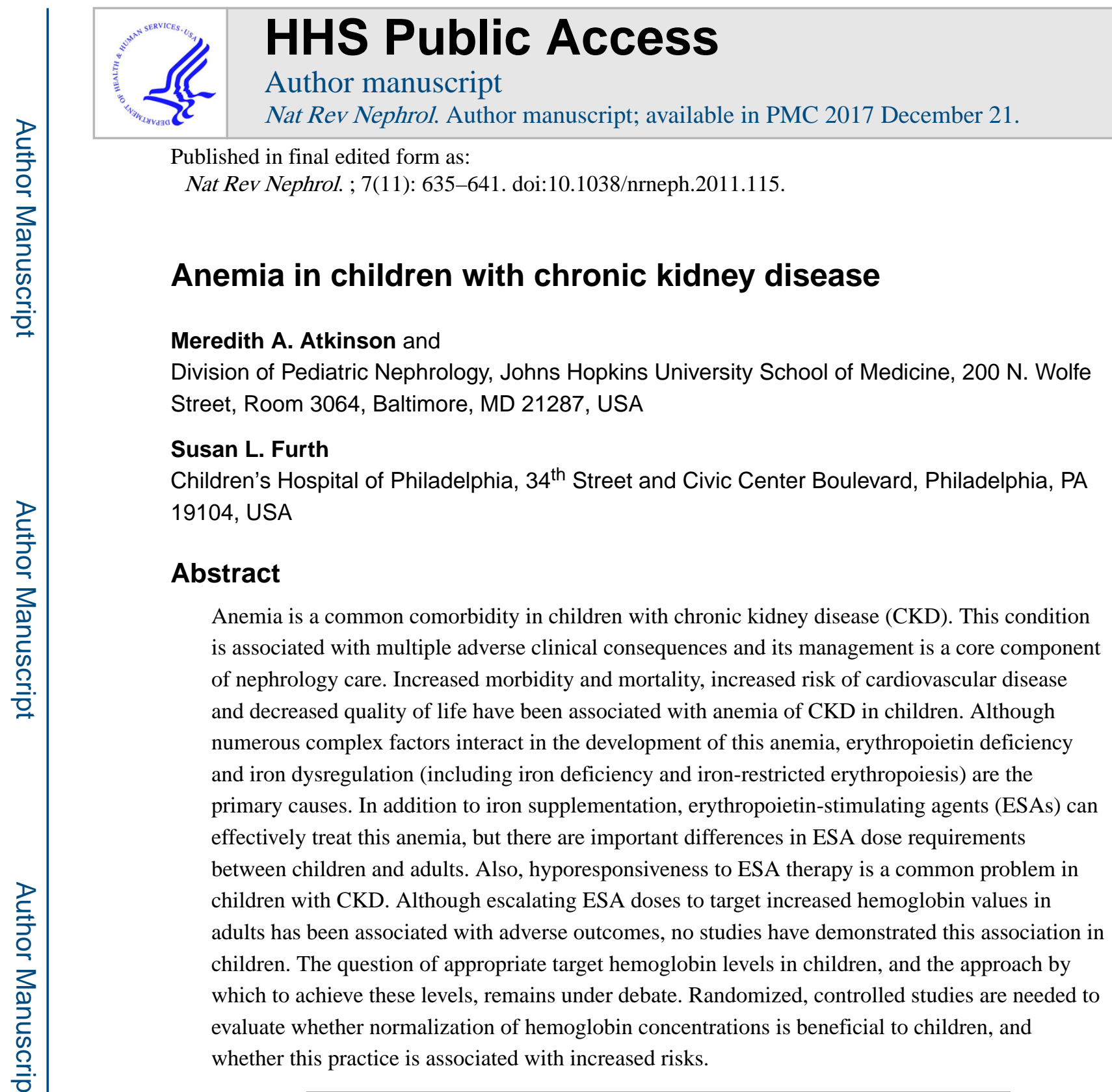

\title{
Introduction
}

A low hemoglobin level is a common comorbidity in children with chronic kidney disease (CKD) and is associated with multiple adverse clinical consequences, including mortality and the development and progression of cardiovascular risk factors, such as left ventricular hypertrophy (LVH). ${ }^{1-3}$ Data from the literature indicate that uncontrolled anemia might be related to the progression of CKD as well as to an increased risk of hospitalization. ${ }^{4-7}$ By contrast, increased hemoglobin values in children with CKD have been associated with improved health-related quality of life. ${ }^{8}$ Thus, anemia management is a core component of

Correspondence to: M. A. Atkinson, matkins3@jhmi.edu.

Competing interests

The authors declare no competing interests.

Author contributions

M. A. Atkinson and S. L. Furth contributed equally to discussion of content for the article, researching data to include in the manuscript and reviewing and editing of the manuscript before submission. 
clinical pediatric nephrology practice. Although many factors, such as shortened lifespan of red blood cells and iron deficiency, contribute to CKD-associated anemia, erythropoietin deficiency is the primary etiological factor.

Defining anemia in children with CKD is not straightforward and requires nuanced evaluation. Although normal hemoglobin values in both adults and children are known to vary based on age, race and sex, anemia management guidelines from the National Kidney Foundation Kidney Disease Outcomes Quality Initiative (NKF-KDOQI) have not consistently recognized these demographic differences with regard to the evaluation and management of the anemia of CKD. ${ }^{9-11}$ In adult patients with CKD, a fixed cut-off hemoglobin value of $<110 \mathrm{~g} / \mathrm{l}$ has been used to define anemia based on evidence that outcomes are worse below this threshold. ${ }^{12}$ Previous versions of the KDOQI anemia guidelines simply applied the adult hemoglobin cut-off to children, but this definition has been shown to substantially underestimate the prevalence of anemia in patients with CKD aged $<18$ years. ${ }^{6}$ Given that children are likely to suffer differently from the effects of various CKD complications, including anemia, compared with adults, the utility of applying an adult hemoglobin treatment target to anemia management in children has been called into question. The most recent NKF-KDOQI clinical practice guidelines use reference data from the National Health and Nutrition Examination Survey (NHANES) III to cite normative values in children, and recommend initiating an evaluation for anemia when hemoglobin levels fall below the age-specific and sex-specific $5^{\text {th }}$ percentile value, although hemoglobin differences by race are still not taken into account (Table 1). ${ }^{12}$ The NHANES III survey data provide means, standard errors and percentile distributions for hematologic laboratory values collected from the US population between 1988 and $1994 .^{9}$

In this Review, we will explore the adverse associations of anemia of CKD in children, with attention to risk factors for its development. The etiological factors for this anemia will be discussed, along with therapeutic strategies and ongoing challenges in its management.

\section{Morbidity and mortality}

In children, as in adults, anemia is a common complication of CKD, which increases in prevalence with advancing stage of CKD. Data from the North American Pediatric Renal Trials and Collaborative Studies (NAPRTCS) have demonstrated that the prevalence of anemia in children is $73 \%$ at stage 3 CKD, $87 \%$ at stage 4 and $>93 \%$ at stage $5 .{ }^{13}$ The anemia of CKD in children is associated with substantial morbidity, an increased risk of cardiovascular disease, decreased quality of life and reduced exercise capacity. Unlike in adults, however, the requirements for linear growth and the increased prevalence of neurocognitive problems in children with CKD might place them at disproportionately increased risk for adverse effects related to persistent anemia. ${ }^{14,15}$ The anemia of CKD has been associated with numerous adverse effects in children. Among adolescents maintained on hemodialysis, decreased hemoglobin levels are a strong and independent predictor of mortality. ${ }^{3,16}$ Anemia has also been associated with increased frequency of hospitalization and increased risk of mortality in children initiating dialysis. ${ }^{17}$ An increased risk of hospitalization in children with anemia has been found not only in those on dialysis, but also among children with predialysis CKD. Staples et al. demonstrated that anemia was 
associated with a nearly $40 \%$ increased risk of all-cause hospitalization over 1 year of follow-up in children with CKD stage 2 and higher. ${ }^{7}$

Anemia is known to increase the risk of developing LVH, which is prevalent in children with CKD and confers an increased risk for cardiovascular disease. ${ }^{2,18}$ This association has been noted not only in children on dialysis, but also among those with CKD stages 2-4. In a cross-sectional analysis of the Chronic Kidney Disease in Children (CKiD) Cohort Study, decreased hemoglobin values were associated with LVH independently of an elevation in blood pressure. ${ }^{18}$ A European cohort study similarly noted an inverse correlation between hemoglobin concentration and left ventricular mass index in patients with early stages of CKD. ${ }^{19}$ Decreased hemoglobin values were also associated with an increased risk of incident $\mathrm{LVH}$ in a longitudinal study of children with CKD. ${ }^{1}$

Increasing evidence shows that anemia may be an independent risk factor for the progression of CKD. A randomized, controlled trial conducted in adult patients with $\mathrm{CKD}$ found that early initiation of erythropoietin therapy in those with mild anemia seemed to delay the initiation of renal replacement therapy. ${ }^{20}$ Furth et al. found that among adolescents (aged 11-18 years) with predialysis CKD, anemia was associated with an accelerated decline in glomerular filtration rate (GFR) compared with the decline rate in patients without anemia. ${ }^{4}$ Data from the NAPRTCS CKD database have shown that anemia, independent of other factors, including underlying diagnosis, hypertension, stage of CKD and race, is associated with an increased risk of CKD progression (defined by reaching CKD stage 5). ${ }^{21}$ Proposed mechanisms include renal tissue hypoxia, which stimulates production of profibrotic cytokines. Results of some erythropoietin trials in adults have suggested that correction of anemia may decrease the risk of CKD progression. ${ }^{4,5}$

Anemia also adversely affects the quality of life of pediatric patients with CKD, including their neurocognitive development and exercise capacity. ${ }^{12}$ Anemic adolescents with CKD have reported greater limitations in physical functioning, schoolwork, and activities with friends and family than their peers who do not have anemia, independent of demographic characteristics including sex, race and level of kidney function. ${ }^{8}$ In terms of growth, a domain in which many children with CKD demonstrate impairment, early treatment of anemia with erythropoietin is associated with improvement. ${ }^{22}$

\section{Pathogenesis of anemia in pediatric CKD}

Although multiple complex factors interact in the development of the anemia of CKD, decreased production of erythropoietin by the diseased kidney is the primary defect (Box 1). In the majority of children with impaired renal function, erythropoietin production is inappropriately low at any given hemoglobin concentration. The mechanism for this decreased production has not been fully elucidated, although it is theorized that it could be a result of progressive loss of the erythropoietin-producing peritubular fibroblast-like interstitial cells in the kidney. ${ }^{23}$ 


\section{Box 1}

Pathogenic factors in the development of anemia in children with CKD

- $\quad$ Erythropoietin deficiency

- Iron-restricted erythropoiesis (e.g. absolute iron deficiency, impaired iron trafficking, functional iron deficiency)

- $\quad$ Nutritional deficiency (e.g. vitamin $\mathrm{B}_{12}$, folic acid, carnitine)

- Uremia

- $\quad$ Bone mineral metabolism disorder

- Inflammation (chronic or acute)

- $\quad$ Chronic blood loss

- $\quad$ Inadequate dialysis

- Medication

- $\quad$ Pure red cell aplasia

Abbreviation: CKD, chronic kidney disease.

Multiple other clinical factors might contribute to anemia among patients with $\mathrm{CKD}$, which worsens with declining GFR. Iron deficiency is clearly associated with anemia, and in children with CKD might be a result of nutritional deficiency, poor enteral absorption of iron supplements, or chronic blood loss from frequent laboratory testing or in the context of chronic hemodialysis. Individual factors that cause anemia in CKD may have varying influence at different levels of GFR. At earlier stages of CKD, nutritional factors and iron deficiency may have a more prominent role in the etiology of anemia. By contrast, decreased production of erythropoietin by the diseased kidney might have a more substantial contribution at later stages of CKD.

\section{Impaired erythropoiesis}

Evaluating the adequacy of iron stores for erythropoiesis is complex in children with CKD. Iron-restricted erythropoiesis interferes with the production of healthy red blood cells by several mechanisms: absolute iron deficiency from inadequate intake or excessive loss, 'functional' iron deficiency (which refers to inadequate storage of iron to supply bone marrow stimulated with erythropoietin-stimulating agents [ESAs]), and impaired iron trafficking in the setting of inflammation. ${ }^{24}$ In order to address this issue clinically, the presence of sufficient iron stores must be documented; in practice, serum levels of ferritin and transferrin saturation (TSAT) are most often used to assess iron availability for erythropoiesis. The KDOQI anemia guidelines recommend that during ESA treatment, children with nondialysis CKD should achieve serum ferritin levels of $>225 \mathrm{pmol} / \mathrm{l}$ and TSAT $>20 \%$, but there are no specific recommendations for target ferritin and transferrin levels for children not receiving treatment with an ESA. ${ }^{12}$ Clear evidence from the literature shows that normal values for iron parameters, including ferritin, transferrin and TSAT, vary 
with age in children, with reference values for all three generally increasing from infancy through adolescence. ${ }^{25,26}$ Although the KDOQI guidelines do recognize age-specific variations in hemoglobin in the recommendations for anemia evaluation, age-specific differences in iron parameters are not considered. Other, potentially more sensitive, indicators of iron status include reticulocyte hemoglobin content, which measures the immediate incorporation of iron into reticulocytes, percentage of hypochromic red cells, defined as red blood cells with cellular hemoglobin concentration $<280 \mathrm{~g} / \mathrm{l}$, and serum soluble transferrin receptor, which is upregulated when iron stores are decreased. ${ }^{25,27,28}$ Although these tests may provide better estimates of iron availability than ferritin or TSAT levels alone, none have attained widespread clinical use.

In clinical practice, serum levels of ferritin are considered to be reflective of iron stores in the body, and TSAT indicative of the amount of iron available to be transported to the bone marrow. TSAT is calculated from serum iron concentration divided by total iron-binding capacity, which is itself an indirect measure of transferrin levels. ${ }^{28}$ However, ferritin and TSAT both have limitations as indicators of iron stores in patients with CKD. Serum ferritin concentration is difficult to interpret as an independent marker of iron status as it is also an acute-phase reactant whose production is upregulated by inflammation. Although serum ferritin concentration is presumed to reflect a steady-state leakage of ferritin stored intracellularly, serum levels may not accurately reflect total iron stores in the body; the process by which intracellular ferritin enters the circulation, from which it is directly measured, is not well understood. ${ }^{28}$ Production of transferrin is also affected by inflammation, although, in contrast to the effect on ferritin, inflammation decreases transferrin production, resulting in misleadingly elevated TSAT values, even in the setting of iron-restricted erythro-poiesis. ${ }^{25,28}$ In children specifically, serum iron levels have been shown to be inversely correlated with BMI z-score, although a clear relationship between BMI and ferritin has not been established. Obesity-associated variations in iron parameters might also be mediated by inflammation. ${ }^{29}$

\section{Inflammation}

Chronic inflammation is common in patients with CKD and is clearly associated with an increased risk of anemia. Proinflammatory cytokines, such as IL-6 and tumor necrosis factor, are associated with anemia and contribute to decreased hematopoiesis by reducing iron availability and inhibiting the maturation of erythroid precursor cells in the bone marrow. ${ }^{30}$ Increased levels of inflammatory markers have been detected in both adults and children with predialysis CKD, well before the initiation of renal replacement therapy. ${ }^{31-33}$ In a study of 64 children with CKD, nearly half had C-reactive protein levels of $>1 \mathrm{mg} / \mathrm{l}$, indicating inflammation. ${ }^{33}$

The anemia of inflammation has long been associated with elevated cytokine levels, but for many years the underlying mechanism of this anemia was not known. The antimicrobial and iron-regulatory peptide hepcidin has emerged as the link between the inflammatory response and the handling of iron for erythropoiesis. Hepcidin is an acute-phase protein produced in the liver and is a negative regulator of iron utilization, which controls blood iron levels both by downregulating the absorption of iron in the intestine and by inhibiting the release of iron 
from iron-storing reticuloendothelial cells. The mechanism for this process is through hepcidin-induced downregulation of ferroportin, which is the major transmembrane iron transporter present on the surface of iron-storing cells. ${ }^{34-36}$ Hepcidin binds directly to ferroportin channels, inducing their internalization and degradation, effectively trapping iron within macrophages, hepatocytes and enterocytes (and reducing intestinal absorption). ${ }^{34,37}$ Iron is thus not made available for erythropoiesis. Hepcidin is freely filtered at the glomerulus and excreted by the kidney, and decreased hepcidin excretion in the context of decreased GFR is likely to contribute to anemia. ${ }^{34}$ In addition, hepcidin has been shown to be directly induced by proinflammatory cytokines, including IL-6, and is positively correlated with levels of C-reactive protein in adult patients with CKD. ${ }^{38-40}$ In a study of 48 children with CKD stages 2-4, 26 children on dialysis and 32 adults with CKD stages 2-4, serum hepcidin levels were compared with those of healthy pediatric and adult controls. Hepcidin levels among children with both end-stage renal disease and earlier stage CKD were significantly higher than levels in the healthy controls $(P<0.001) .{ }^{40}$

\section{Nutritional deficiencies}

Aside from iron deficiency, there are multiple other nutritional deficiencies, including vitamin $B_{12}$, folate and perhaps vitamin $C$, which could interfere with the production of healthy mature red blood cells. Folate deficiency is common in children with CKD, and in a small cohort of children on hemodialysis oral folic acid supplementation resulted in an $11.4 \%$ mean increase in hemoglobin levels and decreased ESA dose requirement. ${ }^{41}$ Carnitine deficiency might also decrease the survival of red blood cells in patients with $\mathrm{CKD}$, as it leads to an increase in the membrane fragility of red blood cells. ${ }^{42}$

\section{Uremia and hyperparathyroidism}

Independent of nutritional factors, chronic uremia may also contribute to anemia. Uremic serum has been shown to shorten the survival of erythrocytes and might also stimulate hemolysis. ${ }^{23,43}$ Hyperparathyroidism has been associated with a poor response to erythropoietin and might be associated with decreased bone marrow production of red blood cells as a result of myelofibrosis..$^{4,45}$ However, although secondary hyperparathyroidism has been associated with anemia in adults, a study of children on hemodialysis could not establish an association between parathyroid hormone levels and the achievement of adult hemoglobin target levels of $110 \mathrm{~g} / 1 .{ }^{46}$

\section{Risk factors}

Multiple risk factors for the development of anemia in children with CKD have been identified, with the level of kidney function being one of the main risk factors. Data from the NAPRTCS cohort have consistently demonstrated that the risk of anemia increases as CKD stage advances. ${ }^{7,13}$ A study performed within the CKiD cohort has shown that anemia is common in pediatric patients with CKD across all stages of CKD, but that hemoglobin levels decline more rapidly as the GFR (determined by plasma disappearance of iohexol) falls below $43 \mathrm{ml} / \mathrm{min} / 1.73 \mathrm{~m}^{2.47}$ Treatment with antihypertensive medications has also emerged as a risk factor for anemia in children with CKD, which may be attributable to the increased risk of anemia conferred by angiotensin-converting-enzyme inhibitors. ${ }^{13,21,48}$ 
Race is also recognized as a risk factor for anemia. Racial disparities in the prevalence of anemia among adults with CKD are well described, ${ }^{49}$ and differences in hemoglobin by race have also been noted in the pediatric CKD population. Among children enrolled in the CKiD study, those of African American descent were noted to have average hemoglobin levels of 6 $\mathrm{g} / \mathrm{l}$ lower than those of white children $(95 \% \mathrm{CI}-9$ to $-2 \mathrm{~g} / \mathrm{l})$, even after adjusting for other relevant characteristics, including GFR, use of iron supplementation and exogenous erythropoietin, and socioeconomic status. ${ }^{11}$

\section{Therapeutic strategies}

Therapy for anemia is a key factor for the reduction of morbidity and mortality in children with CKD. The two primary components of effective anemia management are treatment with an exogenous ESA and supplemental iron therapy. Adjunctive therapies can include treating other nutritional deficiencies and controlling bone mineral metabolism disorders. As recommended by the KDOQI guidelines, primary anemia prevention in children with CKD includes monitoring hemoglobin levels at least annually for detection of anemia, and initiating therapy for absolute iron deficiency as indicated. ${ }^{12}$

\section{Treatment with ESAs}

Recombinant human erythropoietin-Most children with CKD and anemia will ultimately require treatment with an ESA. Recombinant human erythropoietin (rHuEPO), which may be administered intravenously or subcutaneously, effectively treats anemia both in children with predialysis CKD and those on maintenance dialysis. ${ }^{12}$ Epoetin alfa is one of the most commonly used forms of rHuEPO in the USA, with epoetin beta more commonly used in Europe. Multiple other erythropoietin analogues are emerging and the pediatric nephrologist must be constantly attuned to issues of safety and efficacy and how these agents act in children compared with adults. ${ }^{50}$ In addition, it is important to note that the dosing requirements may differ substantially between children and adults. In clinical practice, the rHuEPO 'starting dose' of $150 \mathrm{U} / \mathrm{kg}$ per week is based on data from an adult clinical trial. ${ }^{51}$ Data from NAPRTCS have demonstrated that young children require higher rHuEPO doses than adults, ranging from $275 \mathrm{U} / \mathrm{kg}$ to $350 \mathrm{U} / \mathrm{kg}$ per week for infants, to $200-250 \mathrm{U} / \mathrm{kg}$ per week for older children. ${ }^{45}$ An analysis performed by Bamgbola et al. among patients on chronic hemodialysis found that children and adolescents required higher absolute doses of rHuEPO than adults to maintain target hemoglobin levels, in spite of the fact that mean body weight was lower in the children. ${ }^{52}$ Unlike the dosing of many drugs in children, which is typically based on body size to account for a decreased volume of distribution in children compared with adults, the rHuEPO doses required for successful achievement of target hemoglobin levels in pediatric patients seems to be independent of weight. ${ }^{52,53}$ Rather than determining rHuEPO doses in children based on body weight, it has been suggested that dosing should be based on hemoglobin deficit. In fact, there is evidence that an absolute $\mathrm{rHuEPO}$ dose of 1,000 $\mathrm{U}$ given intravenously can increase hemoglobin by $0.4 \mathrm{~g} / \mathrm{l}$ both in children and adults. ${ }^{51}$ The underlying mechanisms related to the increased requirement of rHuEPO seen in children have not been fully elucidated, but may be due to an increased presence of nonhematopoietic erythropoietin binding sites (for example, endothelial, kidney, skeletal muscle, brain, heart and retinal cells) in children, which may result in increased 
clearance of the drug. ${ }^{45,54}$ The major function of erythropoietin in these various cell types seems to be inhibition of apoptosis. ${ }^{54}$ In addition, erythropoietin demand in children may be increased during periods of accelerated body growth, which clearly is more of a factor in younger children. ${ }^{51}$

Darbepoetin alfa-Darbepoetin alfa, an erythropoietin analogue with a longer half-life than rHuEPO, is now widely used in children with CKD. The benefit of longer dosing intervals compared with those required for rHuEPO has made subcutaneous darbepoetin alfa an attractive alternative for treating anemia in young children and is likely to improve adherence. Darbepoetin alfa may be administered intravenously or subcutaneously, and while drug clearance, half-life and bioavailability are similar for adults and children regardless of route of administration, the absorption of the drug when given subcutaneously may be more rapid in children than in adults. ${ }^{12}$ A randomized clinical trial conducted by Warady et al. in pediatric patients aged $<18$ years with CKD stages 4 or 5 (both dialysis and non-dialysis CKD) demonstrated that darbepoetin alfa is as safe and effective for the correction of anemia as $\mathrm{rHuEPO}$, with the added advantage of less frequent dosing. ${ }^{55}$

A potential limiting factor in the use of darbepoetin alfa in pediatric patients is the reported discomfort associated with its administration. Schmitt et al. conducted a small randomized, controlled trial in children with end-stage renal disease and demonstrated that subcutaneous injections of darbepoetin alfa were associated with significantly higher pain perception in children than injections of subcutaneous epoetin beta $(P<0.05) .{ }^{56}$ Potential explanations for this difference include differences in the types of buffer utilized in each preparation, with the epoetin beta preparation having a more physiological $\mathrm{pH}$ than darbepoetin alfa. ${ }^{56}$

ESA hyporesponsiveness-Anemia that is hyporesponsive to ESA therapy is prevalent in children with CKD, although clarifying the issue of apparent ESA resistance versus inadequate ESA dosing can be challenging. Within the NAPRTCS cohort study, $>20 \%$ of children with CKD stage 4 have been shown to have persistently low hemoglobin levels while on ESA treatment. ${ }^{13}$ In most centers, the management of ESA hyporesponsiveness involves escalation in ESA dose. ${ }^{52}$ However, despite the absence of direct evidence in the pediatric population, data from cohorts of adults with nondialysis CKD enrolled on the Correction of Hemoglobin and Outcomes in Renal Insufficiency (CHOIR) trial and the Cardiovascular Risk Reduction by Early Anemia Treatment with Epoetin Beta (CREATE) trial have caused concern among pediatric nephrologists regarding the appropriateness of this strategy. In the CHOIR trial, the ongoing escalation of ESA dose to target a hemoglobin level of $135 \mathrm{~g} / \mathrm{l}$ was associated with an increased risk of cardiovascular events and death, with no concurrent improvement in quality of life. ${ }^{57}$ Data from CREATE demonstrated that in adults with CKD stages 3 or 4 , early correction of anemia with subcutaneous epoetin beta did not reduce risk of cardiovascular events, although increased hemoglobin targets were associated with improved physical functioning and quality of life.$^{58}$ Thus, the question of the appropriate target hemoglobin levels in children remains under debate. There are many reasons to advocate the normalization of hemoglobin levels in children with CKD, including optimization of growth, cognitive and cardiovascular function, and quality of life.

Randomized, controlled studies in children with CKD are clearly needed to evaluate whether 
targeting higher (and perhaps normalizing) hemoglobin concentrations is associated with benefits to children, and more specifically whether the practice is associated with increased risks as has been demonstrated in adults. In the absence of such data, current recommendations are to target hemoglobin levels of 100-120 g/l in concordance with the FDA guidelines that apply to adults. ${ }^{59}$

Aside from ESA dosing issues in children, there are patients in whom escalating ESA therapy does not correct hemoglobin levels to even a minimal goal, and these are patients often termed ESA hyporesponsive or 'resistant'. In adults with CKD, factors associated with ESA hyporesponsiveness include iron deficiency, chronic blood loss, chronic inflammation, oxidative stress and possibly hyperparathyroidism. ${ }^{60}$ Bamgbola et al. have demonstrated that clinical factors found to be associated with a poor response to $\mathrm{rHuEPO}$ among children maintained on hemodialysis include chronic inflammation (as measured by the objective malnutrition-inflammation score), nutritional deficits (as measured by normalized protein catabolic rate), inadequate dialysis (as measured by urea reduction ratio and $\mathrm{Kt} / \mathrm{V}$ ), chronic blood loss and hyperparathyroidism. ${ }^{52}$ Interestingly, age and sex were not found to be predictive of a poor response to therapy. This observation is in contrast to data in adults with CKD which suggest that older age, use of angiotensin-converting-enzyme inhibitors and angiotensin-receptor blockers, high BMI and diabetes mellitus are predictors of a poor response. ${ }^{61}$ Finally, pure red cell aplasia, secondary to neutralizing antibodies against erythropoietin, has been observed in patients with CKD treated with exogenous erythropoietin, and should be suspected in cases where hemoglobin levels are not responsive to increasing ESA dose or iron therapy. Diagnosis requires the detection of antierythropoietin antibodies, which are generally cross-reactive with all forms of endogenous and exogenous erythropoietin, and treatment often involves immunosuppressive therapy. ${ }^{45,62}$

\section{Iron therapy}

Supplemental iron therapy is another key component of the treatment of anemia of CKD in children. In children with CKD, iron deficiency, defects in iron handling and impaired production of erythropoietin are likely to have a substantial role in the development of anemia. However, little is known about iron status among children with nondialysis CKD. Although clinical practice recommendations from KDOQI for the assessment of iron status and treatment targets do exist for children with CKD treated with an ESA, definitions of iron deficiency and distributions of indices of iron stores in children with mild to moderate CKD have not been well described. The KDOQI anemia guidelines recommend that during ESA treatment, children with nondialysis CKD achieve serum ferritin levels of $>225 \mathrm{pmol} / \mathrm{l}$ and TSAT $>20 \% .{ }^{12}$ However, there are no specific recommendations for target ferritin and TSAT levels for children not receiving treatment with an ESA. Patients with CKD often have normal or above-normal ferritin values, low TSAT levels and persistent anemia, which might indicate inflammation-mediated iron-restricted erythropoiesis. The normal or elevated ferritin value might not reflect total body iron stores that can be utilized appropriately for erythropoiesis, but might instead be a marker of inflammation.

Oral iron supplementation, although inexpensive and readily available, has limited efficacy for maintaining adequate iron stores for erythropoiesis owing to poor gastrointestinal 
absorption and issues of compliance. In addition, oral iron supplementation is often inadequate to keep up with chronic blood loss for children maintained on hemodialysis. Studies in children have confirmed the efficacy of intravenous iron preparations in children on hemodialysis in terms of improving measured iron stores, increasing serum hemoglobin levels and decreasing the dose of ESA required to maintain hemoglobin. ${ }^{42,63}$ In addition, administration of intravenous iron has been shown to result in increased hemoglobin levels in children with nondialysis CKD not maintained on an ESA. ${ }^{64}$ Despite the benefits of intravenous iron, iron overload remains a considerable concern, with nephrologists often hesitating to administer additional iron to patients with serum ferritin levels >1124-1798 $\mathrm{pmol} / \mathrm{l}$. Indeed, iron overload states might be associated with multiple adverse clinical effects in addition to causing increased oxidative stress. ${ }^{65,66}$ Thus, escalation of iron therapy in an attempt to overcome ESA hyporesponsiveness has practical limitations.

\section{Conclusions}

Anemia is prevalent among children with CKD, and is associated with multiple adverse outcomes. Erythropoietin deficiency and iron deficiency are the major underlying etiological factors, and are the mechanisms most likely to be targeted by current therapies. However, assessing the iron status of patients can be challenging, especially in the setting of inflammation and iron-restricted erythropoiesis. With regard to questions concerning the safety and efficacy of escalating ESA doses in response to refractory anemia, there is a need for the identification of pathways beyond erythropoietin deficiency for the development of other safe, nontoxic clinical interventions that could serve to adjunctively treat the anemia in children with CKD.

\section{References}

1. Mitsnefes MM, et al. Progression of left ventricular hypertrophy in children with early chronic kidney disease: 2-year follow-up study. J Pediatr. 2006; 149:671-675. [PubMed: 17095341]

2. Schaefer F. Cardiac disease in children with mild-to-moderate chronic kidney disease. Curr Opin Nephrol Hypertens. 2008; 17:292-297. [PubMed: 18408481]

3. Amaral S, et al. Association of mortality and hospitalization with achievement of adult hemoglobin targets in adolescents maintained on hemodialysis. J Am Soc Nephrol. 2006; 17:2878-2885. [PubMed: 16943308]

4. Furth SL, et al. The association of anemia and hypoalbuminemia with accelerated decline in GFR among adolescents with chronic kidney disease. Pediatr Nephrol. 2007; 22:265-271. [PubMed: 17120062]

5. Wühl E, Schaefer F. Therapeutic strategies to slow chronic kidney disease progression. Pediatr Nephrol. 2008; 23:705-716. [PubMed: 18335252]

6. Filler G, Mylrea K, Feber J, Wong H. How to define anemia in children with chronic kidney disease? Pediatr Nephrol. 2007; 22:702-707. [PubMed: 17216248]

7. Staples AO, et al. Anemia and risk of hospitalization in pediatric chronic kidney disease. Clin J Am Soc Nephrol. 2009; 4:48-56. [PubMed: 19056614]

8. Gerson A, et al. Anemia and health-related quality of life in adolescents with chronic kidney disease. Am J Kidney Dis. 2004; 44:1017-1023. [PubMed: 15558522]

9. Hollowell JG, et al. Hematological and iron-related analytes - reference data for persons aged 1 year and over: United States, 1988-94. Vital Health Stat 11. 2005; 247:1-156. 
10. Jackson RT. Separate hemoglobin standards for blacks and whites: a critical review of the case for separate and unequal hemoglobin standards. Med Hypotheses. 1990; 32:181-189. [PubMed: 2398834]

11. Atkinson MA, et al. Hemoglobin differences by race in children with CKD. Am J Kidney Dis. 2010; 55:1009-1017. [PubMed: 20418001]

12. KDOQI: National Kidney Foundation. KDOQI clinical practice guidelines and clinical practice recommendations for anemia in chronic kidney disease. Am J Kidney Dis. 2006; 47(Suppl 3):S11S145. [PubMed: 16678659]

13. Atkinson MA, Martz K, Warady BA, Neu AM. Risk for anemia in pediatric chronic kidney disease patients: a report of NAPRTCS. Pediatr Nephrol. 2010; 25:1699-1706. [PubMed: 20464428]

14. Slickers J, Duquette P, Hooper S, Gipson D. Clinical predictors of neurocognitive deficits in children with chronic kidney disease. Pediatr Nephrol. 2007; 22:565-572. [PubMed: 17180361]

15. Seikaly MG, Salhab N, Gipson D, Yiu V, Stablein D. Stature in children with chronic kidney disease: analysis of NAPRTCS database. Pediatr Nephrol. 2006; 21:793-799. [PubMed: 16583244]

16. Amaral S, et al. Serum albumin level and risk for mortality and hospitalization in adolescents on hemodialysis. Clin J Am Soc Nephrol. 2008; 3:759-767. [PubMed: 18287254]

17. Warady BA, Ho M. Morbidity and mortality in children with anemia at initiation of dialysis. Pediatr Nephrol. 2003; 18:1055-1062. [PubMed: 12883982]

18. Mitsnefes M, et al. Masked hypertension associates with left ventricular hypertrophy in children with CKD. J Am Soc Nephrol. 2010; 21:137-144. [PubMed: 19917781]

19. Matteucci MC, et al. for the Escape Trial Group. Left ventricular geometry in children with mild to moderate chronic renal insufficiency. J Am Soc Nephrol. 2006; 17:218-226. [PubMed: 16280471]

20. Gouva C, Nikolopoulos P, Ioannidis JP, Siamopoulos KC. Treating anemia early in renal failure patients slows the decline of renal function: a randomized controlled trial. Kidney Int. 2004; 66:753-760. [PubMed: 15253730]

21. Staples AO, et al. Association between clinical risk factors and progression of chronic kidney disease in children. Clin J Am Soc Nephrol. 2010; 5:2172-2179. [PubMed: 20813855]

22. Boehm M. Early erythropoietin therapy is associated with improved growth in children with chronic kidney disease. Pediatr Nephrol. 2007; 22:1189-1193. [PubMed: 17394020]

23. Nangaku M, Eckardt KU. Pathogenesis of renal anemia. Semin Nephrol. 2006; 26:261-268. [PubMed: 16949463]

24. Goodnough LT, Nemeth E, Ganz T. Detection, evaluation, and management of iron-restricted erythropoiesis. Blood. 2010; 116:4754-4761. [PubMed: 20826717]

25. Ooi CL, Lepage N, Nieuwenhuys E, Sharma AP, Filler G. Pediatric reference intervals for soluble transferrin receptor and transferrin receptor-ferritin index. World J Pediatr. 2009; 5:122-126. [PubMed: 19718534]

26. Looker AC, Dallman PR, Carroll MD, Gunter EW, Johnson CL. Prevalence of iron deficiency in the United States. JAMA. 1997; 277:973-976. [PubMed: 9091669]

27. Braun J, Lindner K, Schreiber M, Heidler RA, Hörl WH. Percentage of hypochromic red blood cells as predictor of erythropoietic and iron response after $\mathrm{i} . \mathrm{v}$ iron supplementation in maintenance haemodialysis patients. Nephrol Dial Transplant. 1997; 12:1173-1181. [PubMed: 9198047]

28. Kalantar-Zadeh K, Kalantar-Zadeh K, Lee GH. The fascinating but deceptive ferritin: to measure it or not to measure it in chronic kidney disease? Clin J Am Soc Nephrol. 2006; 1(Suppl 1):S9-S18. [PubMed: 17699375]

29. Sharma AP, McKenna AM, Lepage N, Nieuwenhuys E, Filler G. Relationships among serum iron, inflammation, and body mass index in children. Adv Pediatr. 2009; 56:135-144. [PubMed: 19968946]

30. Girndt M, et al. Influence of cytokine gene polymorphisms on erythropoetin dose requirements in chronic haemodialysis patients. Nephrol Dial Transplant. 2007; 22:3586-3592. [PubMed: 17875573]

31. Goldstein SL, Leung JC, Silverstein DM. Pro- and anti-inflammatory cytokines in chronic pediatric dialysis patients: effect of aspirin. Clin J Am Soc Nephrol. 2006; 1:979-986. [PubMed: 17699316] 
32. Pecoits-Filho R, Sylvestre LC, Stenvinkel P. Chronic kidney disease and inflammation in pediatric patients: from bench to playground. Pediatr Nephrol. 2005; 20:714-720. [PubMed: 15856313]

33. Sylvestre LC, et al. The malnutrition and inflammation axis in pediatric patients with chronic kidney disease. Pediatr Nephrol. 2007; 22:864-873. [PubMed: 17340148]

34. Malyszko J, Mysliwiec M. Hepcidin in anemia and inflammation in chronic kidney disease. Kidney Blood Press Res. 2007; 30:15-30. [PubMed: 17215586]

35. Roy CN, Andrews NC. Anemia of inflammation: the hepcidin link. Curr Opin Hematol. 2005; 12:107-111. [PubMed: 15725899]

36. Swinkels DW, Wetzels JF. Hepcidin: a new tool in the management of anaemia in patients with chronic kidney disease? Nephrol Dial Transplant. 2008; 23:2450-2453. [PubMed: 18495744]

37. Kemna EH, Tjalsma H, Willems HL, Swinkels DW. Hepcidin: from discovery to differential diagnosis. Haematologica. 2008; 93:90-97. [PubMed: 18166790]

38. Means RT. Hepcidin and cytokines in anaemia. Hematology. 2004; 9:357-362. [PubMed: 15763974]

39. Nemeth E. Targeting the hepcidin-ferroportin axis in the diagnosis and treatment of anemias. Adv Hematol. 2010; 2010:750643. [PubMed: 20066043]

40. Zaritsky J, et al. Hepcidin-a potential novel biomarker for iron status in chronic kidney disease. Clin J Am Soc Nephrol. 2009; 4:1051-1056. [PubMed: 19406957]

41. Bamgbola OF, Kaskel F. Role of folate deficiency on erythropoietin resistance in pediatric and adolescent patients on chronic dialysis. Pediatr Nephrol. 2005; 20:1622-1629. [PubMed: 16133045]

42. Greenbaum LA. Anemia in children with chronic kidney disease. Adv Chronic Kidney Dis. 2005; 12:385-396. [PubMed: 16198278]

43. Kruse A, Uehlinger DE, Gotch F, Kotanko P, Levin NW. Red blood cell lifespan, erythropoiesis and hemoglobin control. Contrib Nephrol. 2008; 161:247-254. [PubMed: 18451684]

44. Rao DS, Shih MS, Mohini R. Effect of serum parathyroid hormone and bone marrow fibrosis on the response to erythropoietin in uremia. N Engl J Med. 1993; 328:171-175. [PubMed: 8417383]

45. Koshy SM, Geary DF. Anemia in children with chronic kidney disease. Pediatr Nephrol. 2008; 23:209-219. [PubMed: 17245602]

46. Smith LB, et al. Secondary hyperparathyroidism and anemia in children treated by hemodialysis. Am J Kidney Dis. 2010; 55:326-334. [PubMed: 20116689]

47. Fadrowski JJ, et al. Hemoglobin decline in children with chronic kidney disease: baseline results from the Chronic Kidney Disease in Children Prospective Cohort Study. Clin J Am Soc Nephrol. 2008; 3:457-462. [PubMed: 18235140]

48. Cruzado JM, Rico J, Grinyó JM. The renin angiotensin system blockade in kidney transplantation: pros and cons. Transpl Int. 2008; 21:304-313. [PubMed: 18266774]

49. Collins AJ, et al. Excerpts from the United States Renal Data System 2007 annual data report. Am J Kidney Dis. 2008; 51(Suppl 1):S1-S320.

50. Covic A, et al. Biosimilars and biopharmaceuticals: what the nephrologists need to know-a position paper by the ERA-EDTA Council. Nephrol Dial Transplant. 2008; 23:3731-3737. [PubMed: 18802209]

51. Port RE, Mehls O. Erythropoietin dosing in children with chronic kidney disease: based on body size or on hemoglobin deficit? Pediatr Nephrol. 2009; 24:435-437. [PubMed: 18696118]

52. Bamgbola OF, Kaskel FJ, Coco M. Analyses of age, gender and other risk factors of erythropoietin resistance in pediatric and adult dialysis cohorts. Pediatr Nephrol. 2009; 24:571-579. [PubMed: 18800231]

53. Port RE, Kiepe D, Van Guilder M, Jelliffe RW, Mehls O. Recombinant human erythropoietin for the treatment of renal anaemia in children: no justification for bodyweight-adjusted dosage. Clin Pharmacokinet. 2004; 43:57-70. [PubMed: 14715051]

54. Foley RN. Erythropoietin: physiology and molecular mechanisms. Heart Fail Rev. 2008; 13:405414. [PubMed: 18236154] 
55. Warady BA, Arar MY, Lerner G, Nakanishi AM, Stehman-Breen C. Darbepoetin alfa for the treatment of anemia in pediatric patients with chronic kidney disease. Pediatr Nephrol. 2006; 21:1144-1152. [PubMed: 16724235]

56. Schmitt CP, Nau B, Brummer C, Rosenkranz J, Schaefer F. Increased injection pain with darbepoetin-alpha compared to epoetin-beta in paediatric dialysis patients. Nephrol Dial Transplant. 2006; 21:3520-3524. [PubMed: 16935904]

57. Singh AK, et al. for the CHOIR Investigators. Correction of anemia with epoetin alfa in chronic kidney disease. N Engl J Med. 2006; 355:2085-2098. [PubMed: 17108343]

58. Drüeke TB, et al. Normalization of hemoglobin level in patients with chronic kidney disease and anemia. N Engl J Med. 2006; 355:2071-2084. [PubMed: 17108342]

59. Keithi-Reddy SR, Singh AK. Hemoglobin target in chronic kidney disease: a pediatric perspective. Pediatr Nephrol. 2009; 24:431-434. [PubMed: 18604563]

60. Kwack C, Balakrishnan VS. Managing erythropoietin hyporesponsiveness. Semin Dial. 2006; 19:146-151. [PubMed: 16551293]

61. Rossert J, Gassmann-Mayer C, Frei D, McClellan W. Prevalence and predictors of epoetin hyporesponsiveness in chronic kidney disease patients. Nephrol Dial Transplant. 2007; 22:794800. [PubMed: 17210593]

62. Bennett CL, et al. Long-term outcome of individuals with pure red cell aplasia and antierythropoietin antibodies in patients treated with recombinant epoetin: a follow-up report from the Research on Adverse Drug Events and Reports (RADAR) Project. Blood. 2005; 106:33433347. [PubMed: 16099877]

63. Morgan HE, Gautam M, Geary DF. Maintenance intravenous iron therapy in pediatric hemodialysis patients. Pediatr Nephrol. 2001; 16:779-783. [PubMed: 11605781]

64. Morgan HE, Holt RC, Jones CA, Judd BA. Intravenous iron treatment in paediatric chronic kidney disease patients not on erythropoietin. Pediatr Nephrol. 2007; 22:1963-1965. [PubMed: 17704954]

65. Agarwal R. Iron, oxidative stress, and clinical outcomes. Pediatr Nephrol. 2008; 23:1195-1199. [PubMed: 18030498]

66. Fishbane S. Upper limit of serum ferritin: misinterpretation of the 2006 KDOQI anemia guidelines. Semin Dial. 2008; 21:217-220. [PubMed: 18248518] 


\section{Review criteria}

Material for this Review was obtained by searching PubMed using the following terms (alone and in various combinations): "kidney failure, chronic", "end-stage kidney disease", "chronic", "kidney", "anemia", "hemoglobin", "pathogenesis", "risk factors", "mechanism", "treatment", "erythropoiesis-stimulating agents", "EPO", "recombinant human erythropoietin", "darbepoetin", "children", "pediatric", "morbidity", "mortality", "progression", "hospitalization", "left ventricular hypertrophy", "growth", "iron deficiency", "ferritin", "inflammation" and "hepcidin". Selected materials were fulllength, English-language papers, with a focus on studies of individuals aged $<18$ years and on papers published since 2000. The reference lists of identified papers were searched for further material. 


\section{Key points}

- Anemia, in particular anemia that is poorly responsive to treatment, is very common in children with chronic kidney disease (CKD) and end-stage renal disease

- Adverse effects of anemia in this population include the development of left ventricular hypertrophy, increased risk of hospitalization and mortality, progression of kidney disease and decreased quality of life

- Iron-restricted erythropoiesis has a key role in the development of CKDassociated anemia and is mediated in part by inflammation and the ironregulatory protein hepcidin

- $\quad$ Erythropoietin-stimulating agents (ESAs) and iron supplementation remain the mainstays of therapy for the anemia of CKD in children, with children demonstrating higher ESA dosing requirements than adults

- The question of the appropriate target hemoglobin level in children remains under debate, and randomized, controlled studies are needed in children with CKD to evaluate the risks and benefits of targeting normalization of hemoglobin levels

- $\quad$ Regarding the safety and efficacy of escalating ESA doses, the identification of pathways beyond erythropoietin deficiency is needed to develop other safe, nontoxic clinical interventions to treat the anemia of CKD 


\section{Table 1}

$5^{\text {th }}$ percentile hemoglobin values in children ${ }^{12}$

\begin{tabular}{|lll|}
\hline \multirow{2}{*}{ Age (years) } & $\mathbf{5}^{\text {th }}$ percentile for hemoglobin value (g/l) \\
\cline { 2 - 3 } & Females & Males \\
\hline $1-2$ & 108 & 107 \\
\hline $3-5$ & 111 & 112 \\
\hline $6-8$ & 115 & 115 \\
\hline $9-11$ & 119 & 120 \\
\hline $12-14$ & 117 & 124 \\
\hline $15-19$ & 115 & 135 \\
\hline
\end{tabular}

